Chapter 4

\title{
HPV L1 Detection as a Prognostic Marker for Management of HPV High Risk Positive Abnormal Pap Smears
}

\author{
Ralf Hilfrich \\ Additional information is available at the end of the chapter
}

http://dx.doi.org/10.5772/55902

\section{Introduction}

\subsection{Cervical cancer and HPV}

Cervical cancer is still the $2^{\text {nd }}$ most common cancer in women worldwide, and especially women in developing countries are suffering from the disease [1].

If detected at an early stage Cervical Cancer is preventable. In almost all places around the world the traditional Pap smear is used as primary screening tool and even from being far away to be a perfect test, the benefits of Papanicolaou based cervical cancer screening programs in reducing morbidity and mortality are well accepted.

About 50 years after Papanicolaou has started his initial work, Meisels \& Fortin published in 1976 a report in which they showed that the "halo cell" in pap smears, was a koilocyte - the pathognomic cell of an HPV infection [2] and that low grade or mild dysplasia of the cervix had the histological features of a papillomavirus infection [3].

Shortly thereafter Lutz Gissmann in the zur Hausen laboratory in Erlangen cloned from genital warts 'condyloma acuminata', a novel HPV DNA classified as HPV6 [4].

The idea that Cervical Cancer and cancers at other sides could be caused by a viral infection developed afterwards step by step.

In the meantime both concepts are merged and it is accepted that a persisting infection caused by the sexual transmitted human papillomavirus (HPV), is almost always the trigger for the occurrence of cervical cancer and the main agent for cervical epithelial dysplasias. 
Of the more than $120 \mathrm{HPV}$ subtypes known today, the anogenital ones are further divided into low risk (LR-HPV) and currently at least 15 high risk types (HR-HPV; HPV 16, 18, 45, 31, 33, 52,58 are most frequent). While the former are only in rare cases cancerogenic, HR-HPV are detected virtually regularly in high-grade intraepithelial neoplasias and invasive cervical cancers, of which more than 70\% are HPV 16 and HPV 18 [5].

The negative predictive value of the highly sensitive DNA determination of HR-HPV in the cervical smear cell material is with more than $99 \%$ very high, i.e. women not infected with these HPV subtypes very probably have no high-grade intraepithelial lesion and no cervical cancer.

On the other hand the use as primary screening tool for cervical cancer is limited because the positive predictive value of a HPV DNA test is low.

The majority of HR-HPV infections remain morphologically undetected and even in mild dysplasias with persisting infections the probability for the development of high grade intraepithelial lesions is only about $20 \%$ focusing on young women.

Therefore even if thinking about the possibility to replace cytology as primary screening tool, the Pap-test will remain to verify a positive HPV DNA test, to confirm the presence of abnormal cells and most important to determine the severity or grade of disease, a unique feature of the morphological methods.

\section{Cervical cancer screening}

Since the early days of cervical cytology it is known that the morphologically identified lesions of different grades are mixtures of distinct biological stages resulting in different clinical outcomes, remission or progression [6].

Today with the knowledge that HPV is a necessary but not sufficient cause for the development of most cervical cancers, transient HPV infection and precancer are often used synonymous for these biologically different conditions.

Traditionally cervical cancer prevention programs rely on the repeated application of a 3-step strategy:

1. Screening by cervical cytology with the Pap - Test;

2. Follow up of the cytology positive women with HPV DNA testing colposcopic evaluation and directed biopsy of abnormal-looking cervical tissue for diagnosis; and if necessary

3. Excisional or ablative treatment of the cervical tissue in women diagnosed with CIN2+ or precancer.

For Pap smear diagnosis different reporting systems are in use worldwide. Besides the original WHO classification [7], The Bethesda System (TBS) [8] is internationally accepted, while the Munich Nomenclature II is being recommended in Germany [9]. 
Based on Ostors meta-analysis it's common sense that treatment isn't warranted for early dysplastic lesions as for atypical squamous cells of undetermined significance (ASCUS) and mild dysplasia (LSIL) since only $10 \%$ of the mild dysplastic lesions will progress to CIN3+ [10].

A cytological follow up with colposcopic evaluation is recommended for these women and if necessary directed biopsies of abnormal-looking cervical tissue for diagnosis are taken.

The cervical histopathologic diagnoses of these samples are graded according to the cervical intraepithelial neoplasia (CIN) system as normal, CIN1, CIN2, CIN3, and cervical cancer [11].

So far diagnosis of CIN2 or worse is the clinical threshold leading to ablative or excisional therapy in the United States and Europe.

In the meantime a discussion started if moderate dysplasias, the cytological CIN2 equivalent, should be called low grade or high grade lesion. Ostor reported that only $20 \%$ of the moderate dysplasitic lesions progressed to CIN3+, typically a threshhold that should not warrant invasive procedures.

As a consequence of the current situation conisations are being performed frequently, with potentially negative impact on reproductive outcomes for fertile women, including preterm delivery and low-birth-weight infants [12] with possible life long fatal disabilities.

Despite the conservative recommendation in the Munich Nomenclature II, not to treat CIN2, conisation was one of the most common surgical procedures performed in women of fertile age in Germany during 2010, accounting for more than 50.000 cases [13].

The dilemma is that neither cytological follow up, colposcopy nor HPV DNA testing could indicate whether a remission or progression of the precursor lesion to invasive cancer will occur. Therefore more specific tools like prognostic markers would be of great value, allowing an individualized management of cervical lesions depending on their risk profil.

\section{HPV L1 detection as prognostic marker for early dysplastic lesions}

Over the last couples of years cytological samples as well as colposcopically guided punch biospsies, have been used to determine if Cytoactiv HPV L1 detection is able to predict the clinical outcome of HPV high risk positive early dysplastic lesions.

Moderate dysplastic lesions, being part of HSIL, have been investigated as well since the Munich nomenclature II, in contrast to TBS, groups moderate dysplastic lesions, together with mild dysplasias, in the category IIID, with recommendation for cytological follow up and colposcopy.

This different risk assesement offered the unique possibility to follow up these women with moderate dysplasias as well. 


\section{The HPV L1 capsid protein and the viral life cycle}

L1 or the major capsid protein is one of eight known HPV specific proteins (E1, E2, E4, E5, E6, E7, L1 and L2).

It is produced within the cytoplasm and translocated into the nucleus, clearly visible by strong nuclear immunochemical staining reaction in intermediate and superficial squamous epithelial cells.

The L1 protein forms an icosahedral capsid with a T=7 symmetry and a $50 \mathrm{~nm}$ diameter. The capsid is composed of $72 \mathrm{~L} 1$ pentamers, linked to each other by disulfide bonds, and associated with the minor capsid protein L2, which encapsulates the viral DNA to build new infectious viral particles that are released in the upper epithelial layer. [14]

At the same time, L1 is a ligand for a still not reliably identified surface receptor, a heparan sulfate proteoglycan, on the basal cell layer of the epithelium to provide initial virion attachment to target cells. As a general rule, the HPV gains access to the basal epithelial layers as a result of epithelial erosions or mucosal ulcerations in the transformation zone susceptible to inflammation at the cervical/endocervical junction.

Once attached, the virion enters the host cell via a L2 dependant, clathrin-mediated endocytosis, the capsid becomes decraded, the virus DNA is released and routed into the nucleus of the cell [15].

The virus genome then separately lies outside the chromosomal DNA of the host cell as a ringshaped, episomal DNA molecule.

These initial steps are not associated with cellular changes that can be detected by morphological methods. This individually variably long so called latent or silent virus infection can only be detected with molecular biological methods.

The signals to leave the latent virus infection and to initiate the productive or permissive phase of the viral life cycle, leading to a L1 synthesis, are not identified yet. Once differentiation of the immature squamous epithelial host cells begins, the viral DNA starts to replicate to high copy numbers. In the further course and dependent on the host cell differentiation the late proteins are synthesized, and encapsidates the viral DNA. Thus, mature, infectious viruses emerge, which are released from the perishing superficial squamous epithelia [16].

Within the scope of this productive phase, morphological epithelial changes mostly occur after several weeks or months post infection, which allow the cytological diagnosis of dysplasia in the smear. The typical morphological changes like nuclear enlargement, multinucleosis, changes in the chromatin structure and cytoplasm composition as well as koilocytes or 'halo cells' have already been described by Papanicolaou.

Upon termination of the productive phase, the viral life cycle from primary infection to the release of the virus is completed without any malignant neoplasia having occurred (Figure 1). The L1 capsid protein is detectable at that stage of the life cycle, only. 


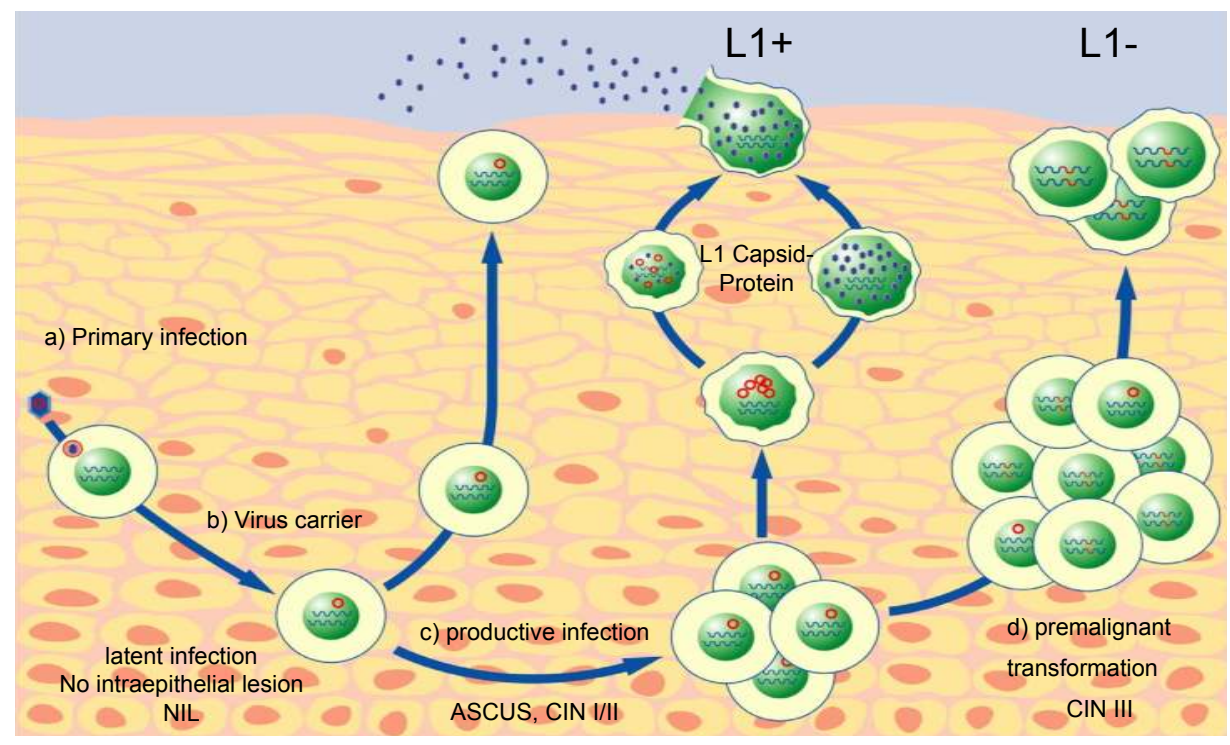

Figure 1. HPV life cycle, as described in the text.

\section{Study results}

A particular methodical advantage of the L1 capsid protein detection is that the protein is synthesized in the cells of the superficial layer of the epithelium that are easy to obtain by taking a smear (see Figure 2).

The typical L1 staining is a strong, homogenous nuclear stain (Figure 3-7), in contrast to other markers, leading to a very good interobserver reproducibility. Using histological sections Galgano et al. [17] reported for Cytoactiv a raw agreement and k of $96.9 \%$ and 0.88 , respectively. With 98\% raw agreement and 0.96 for kappa Mehlhorn et al. [26] reported similar results for the use of Cytoactiv in cytological samples. 


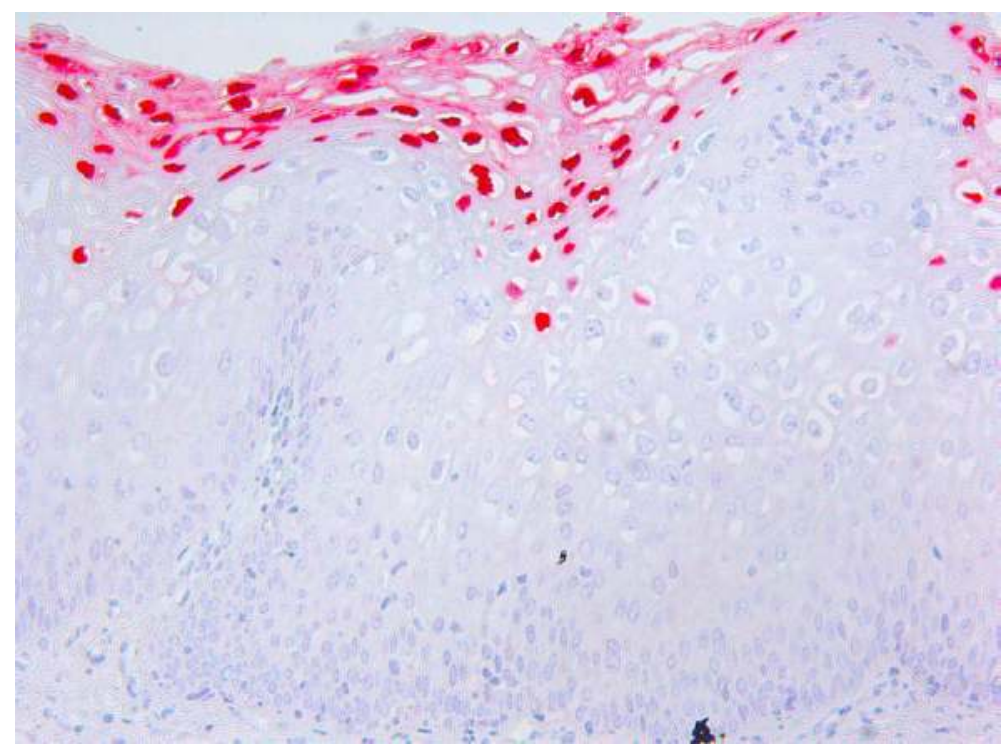

Figure 2. L1+ CIN1 -A particular methodical advantage of the L1 capsid protein detection is that the protein is synthesized in the cells of the superficial layer of the epithelium that are easy to obtain by taking a smear

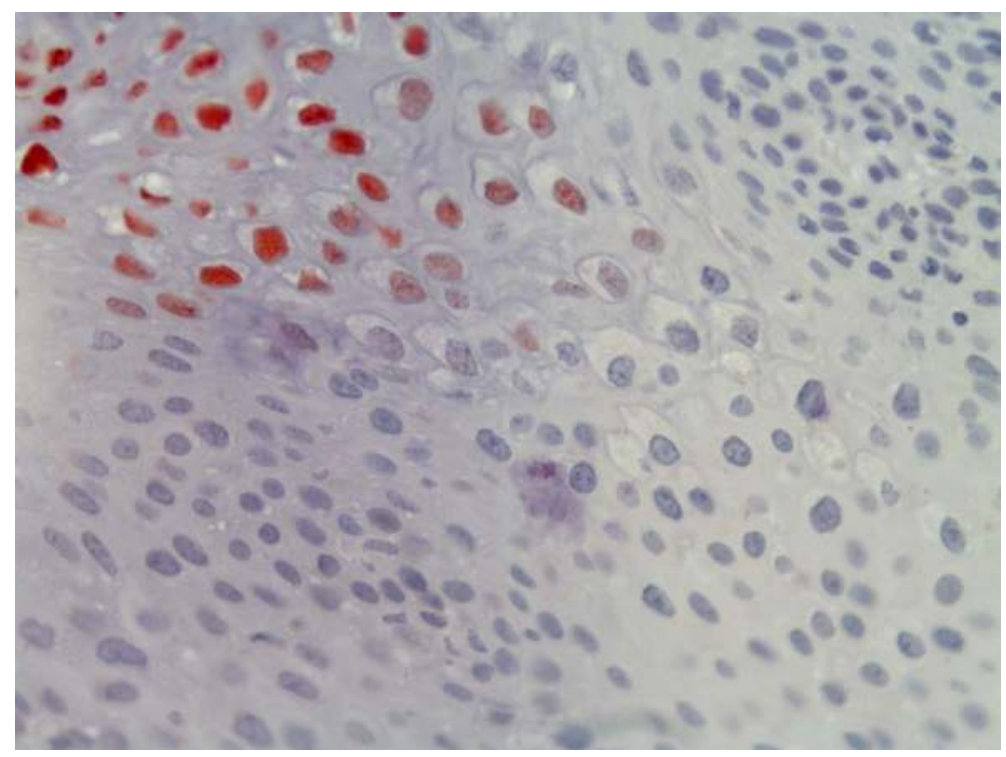

Figure 3. L1 staining intensity correlates with the amount of L1 capsid protein producedand it becomes more intense towards the surface of the epithelium 

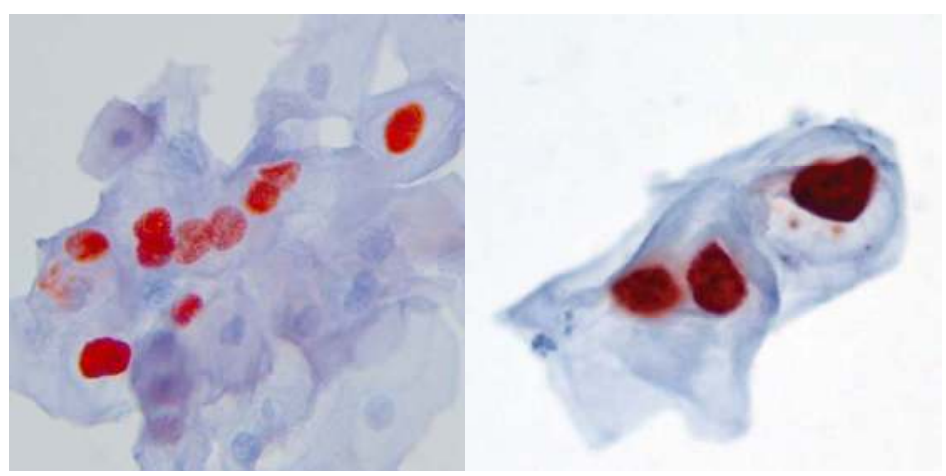

Figure 4. L1 positive LSIL

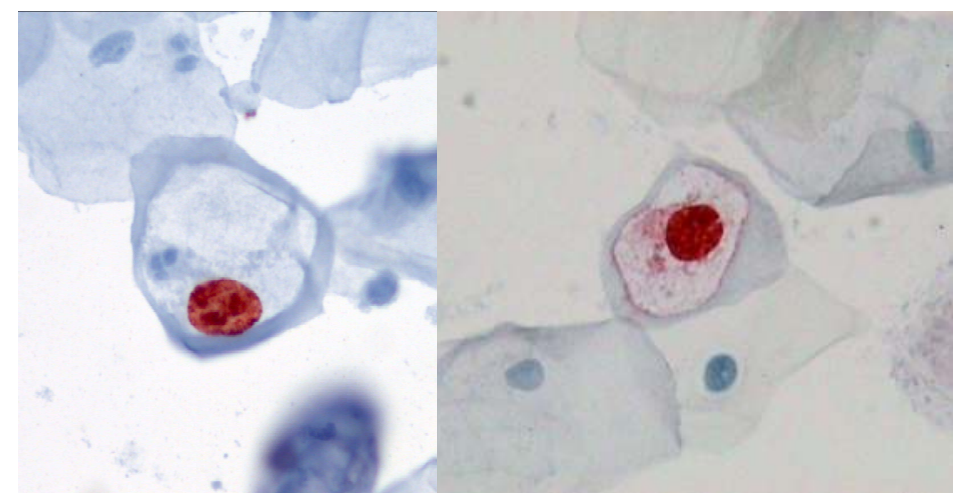

Figure 5. L1 + Koilocytes

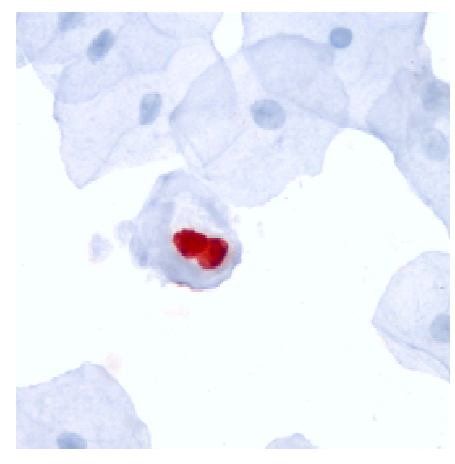

Figure 6. L1 + Koilocyte with 2 nuclei 

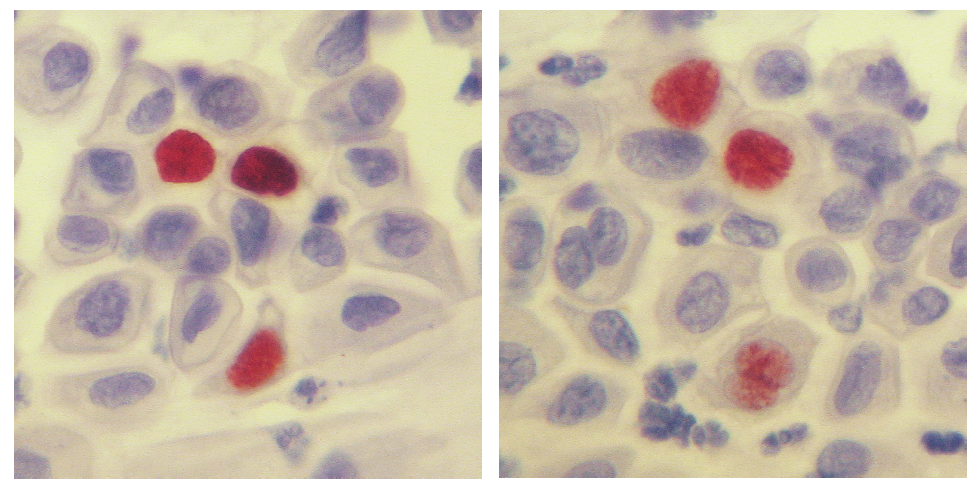

Figure 7. L1+ HSIL, Mehlhorn et al. [26]

Initial L1 studies faced the problem that the sensitivity of randomly choosen L1 antibodies was unacceptable low. One major problem was the well known high frequency of point mutations leading to a loss of the relevant epitopes, and false negative results as a viral strategy to escape immune recognition. This high variability is in clear contrast to the stability of epitopes recognized by antibodies detecting cellular proteins, like p16 or ki67.

During the product development of Cytoactiv it was possible to increase the sensitivity significantly by extensive selection processes generating an optimized antibody detecting a specific epitope.

Nevertheless there were a small number of cases where it was impossible to detect the L1 capsid protein. The reason for this finding was not clear in the beginning.

\subsection{L1 and cytological samples}

In 2003 Melsheimer et al. [18] have published that most of the HPV high risk associated LSIL expressed HPV L1 capsid protein, but in most of the HSIL cases the HPV L1 capsid protein was not synthesized (see Figure 3-6).

They suggested that a loss of viral L1 capsid protein, as a major target of the immune response in HPV infected SIL, could function as a prognostic marker for the development of CIN lesions.

Later on Griesser et al. [19] were able to confirm this suggestion in a retrospective study with 84 routinely performed conventional Pap smears. During a follow up time of 23 month they showed that the HPV high risk associated mild to moderate dysplastic squamous lesions without immunochemically detectable HPV L1 capsid protein progressed significantly more likely to CIN3+ $(76,4 \%)$ than the L1 positive cases $(23,6 \%)$.

Similar results were reported by Rauber et al. [20] in 2008 in a retrospective study of 279 HPV High risk positive conventional Pap smears with mild and moderate severe morphological changes. The progression rate to CIN3+ of L1 capsid protein positive cases was found to be only $12,3 \%$ (p-value $<0,001)$. 
In the same year Scheidemantel et al. [21] tested the Cytoactiv - Kit on 111 HPV High risk positive ThinPrep - slides. They reported that none of the L1 positive patients showed a progression towards cervical cancer and on the other hand all progredient cases were found to be L1 negative.

An additional advantage of choosing the ThinPrep system emerged in the meantime. The ThinPrep Imager allows the automated evaluation of the L1 stained slides, to speed up the reading process [22]. The benefit of computer based automatisation can be extended to conventional Pap smears and SurePath slides as well if choosing BD's focal point system.

The first prospective randomized study was published in 2009 by Griesser and colleagues [23]. The study included 211 HPV High risk-positive mild and moderate dysplasias (LSIL and HSIL) with a follow-up of the patients up to 48 months. The results of all former retrospective studies were confirmed and strengthened. Depending on patients age $(<30 /$ $>30$ ) and the classification of the precancerous lesion (LSIL or HSIL) only $20 \%$ of all L1 positive cases showed a progression to CIN3+. In contrast to this finding up to $97 \%$ of the L1 negative cases showed a progression.

In this study the mean duration from the initial L1 positively / negatively stained smears to the recognition of disease progression or remission were reported as well.

For L1 negative cases the time interval until progression was 6 months (range, 1-29) and 6.4 months (range 2-12) for clinical remission, whereas for the L1 positive cases it took 8.5 months (range, 1-13) until progression, and 7 months (range, 3-35) for clinical remission respectively.

\begin{tabular}{lllllll}
\hline & L1- & L1- LSIL & L1- HSIL & L1+ & L1+ LSIL & L1+ HSIL \\
\hline Remission & $6.4(2-12)$ & $7.5(3-12)$ & 2 & $7(3-35)$ & $6.5(3-18)$ & $9(3-35)$ \\
\hline Progression & $6(1-29)$ & $6(1-29)$ & $6(1-20)$ & $8.5(1-13)$ & $9(6-13)$ & $8(1-13)$ \\
\hline
\end{tabular}

Table 1. Griesser et al., AJCP 2009: Time interval until clinical remission or progression to CIN3 in month (range)

Stemberger - Papic et al. [24] reported similar results for Croatian women and concluded that immunostaining for HPV L1 capsid protein could offer prognostic information about mild and moderate intraepithelial cervical squamous lesions.

In 2010 and 2011 two Korean studies reported that the prognostic significance of the L1 detection with Cytoactiv for the clinical outcome of early dysplastic lesions can be confirmed for East Asian women as well.

Lee et al [25] confirmed 2011 in a prospective trial of 318 women the benefit for Cytoactiv for the management of HPV high risk positive LSIL women. The positive predictive value of HPV L1-positive cases for no progression was $91.7 \%$, and the negative predictive value of HPV L1negative cases for progression to high-grade lesions was 27.7.

The results of the largest study so far, a prospective international multicenter study of $809 \mathrm{HPV}$ High risk positive LSIL and HSIL was performed by Mehlhorn et al. [26]. 
During the follow up of 54 month $83,5 \%$ of the HPV-L1 negative progressed to CIN3+, as compared to only $19,8 \%$ of the HPV-L1 positive cases. The difference of the clinical outcome of HPV-L1 negative and HPV-L1 positive cases was statistically highly significant ( $\mathrm{p}$-value $<0.0001$ ) and independent of the classification as mild dysplasia (LSIL) and moderate dysplasia (HSIL).

The authors concluded that HPV-L1 detection allows identifying transient HPV infections and precancerous lesions within the group of HPV high-risk positive early dysplastic lesions.

The high progression rate of HPV-L1 negative mild and moderate dysplasia emphasizes the precancerous nature of these lesions.

As a clinical recommendation they suggested that a close follow-up with colposcopy and histological evaluation and removal of these lesions should be considered.

The low malignant potential of HPV-L1 positive cases, however, indicates transient HPV infection, justifying a watch and wait strategy with cytological follow-up thus preventing overtreatment especially for women in their reproductive age.

\begin{tabular}{lcccccc}
\hline \multicolumn{1}{c}{ Author } & classification & No.cases & L1 negative & L1 positive & Mean age & Follow up \\
\hline Mehlhorn & LSIL & 479 & 72,9 & 11,8 & 33,6 & 54 month \\
\hline Griesser & LSIL & 68 & 84 & 25 & 33,6 & 48 month \\
\hline in total & & 547 & 75 & 13,1 & 33,6 & \\
\hline
\end{tabular}

Table 2. Risk profil LSIL - Progression to CIN3+ for L1+ and L1- cases

\begin{tabular}{lcccccc}
\hline \multicolumn{1}{c}{ Author } & classification & No.cases & L1 negative & L1 positive & Mean age & Follow up \\
\hline Mehlhorn & HSIL & 322 & 92,7 & 37,4 & 33,6 & 54 month \\
\hline Griesser & HSIL & 119 & 96,9 & 33 & 33,6 & 48 month \\
\hline in total & & 441 & 94,2 & 34,6 & 33,6 & \\
\hline
\end{tabular}

Table 3. Risk profil HSIL - Progression to CIN3+ for L1+ and L1- cases

\section{2. $\mathrm{L} 1$ and histological sections}

As already mentioned earlier colposcopically guided punch biopsies are taken during the follow up of women with abnormal Pap smears as step 2 of the 3 step strategy of cervical cancer prevention.

Negri and colleagues [27] pointed out in their study that the possibility of predicting the behavior of low-grade cervical lesions could be of high value in clinical practice, potentially allowing an individualized management of cervical lesions depending on their progression risk. 
Hilfrich and Hariri [28] have discribed first, the prognostic relevance of HPV L1 capsid protein detection on paraffin embedded histological sections, initially reported on routinely performed Papanicolaou stained cervical smears and on liquid based cytology (LBC) [18], [19] (see Figure 9).

In contrast to these cytology reports, the association of the cervical intraepithelial lesions with HPV high risk types was not confirmed by highly sophistic DNA methods like PCR [18] or Hybrid capture II [19], but the use of a second biomarker, p16, which together with L1, can be easily integrated in any histopathology lab.

Overall only $16.1 \%$ of the $87 \mathrm{~L} 1$ negative, p16 positive CIN lesions showed a remission of the lesion, compared to $72.4 \%$ of the double positive cases. None of the L1/p16 double negative CIN lesions progressed. Hariri found similar results for the combination of ProExC and Cytoactiv.

Negri and colleagues [27] included in their approach 38 conization specimens with coexisting cervical intraepithelial neoplasia grade 1 (CIN1) and 3 (CIN3) (group A) and 28 punch biopsies from women with CIN1 and proven spontaneous regression in the follow-up (group B). In group A, all CIN3 were $\mathrm{p} 16$ positive (p16+) and L1 negative (L1-). The CIN1 of this group were p16+ L1- and p16+ L1+ in $68.42 \%$ and $31.57 \%$, respectively. No other expression pattern was found in this group. In group B, the p16+ L1-, p16+ L1+, p16- L1+, and p16- L1- patterns were found in $3.57 \%, 25 \%, 14.29 \%$, and $57.14 \%$, respectively. Overall, $96.29 \%$ p16+ L1- CIN1 were found in group A, whereas all the p16- L1+ and p16- L1- CIN1 were found in group B.

They found that no cases with both L1 and p16 negativity were found in group A, and proposed that this pattern might be classified as "low risk" or, unless the original section shows obvious dysplastic features, as "no evidence of CIN.'

The results of the study showed that p16 and L1 immunohistochemistry can be helpful for estimating the biologic potentiality of low-grade squamous cervical lesions. Particularly in cases in which the grade of the lesion is morphologically difficult to assess, the p16/L1 expression pattern could be useful for planning the clinical management of these women.

\begin{tabular}{lll}
\hline Staining pattern & $\begin{array}{l}\text { Risk profile } \\
\text { Negri et al. }\end{array}$ & $\begin{array}{l}\text { Risk profile } \\
\text { Hilfrich / Hariri }\end{array}$ \\
\hline P16+ / L1 - & "high-risk", & High risk \\
& $3,6 \%$ remission & $16.1 \%$ remission \\
\hline P16+ / L1+ & indeterminate" risk & $72,4 \%$ remission \\
& & not distinguished in p16+/- \\
\hline P16- / L1+ & "low-risk" & No potential to progress \\
\hline P16- / L1- & "low risk", or & \\
\hline
\end{tabular}

Table 4. Risk profils according to Negri et al./ Hilfrich, Hariri 
Using 101 HPV High risk positive CIN1 Choi et al [29] published 2010 that the HPV L1 protein expression is closely related to spontaneous disease regression. Not using p16, but a typespecific HPV-DNA Chip, it was possible for the first time to correlate the HPV type with the regression of the L1 positive CIN1 lesions. 50\% of the HPV16 positive CIN1, 72,7\% of the HPV $58,76.9 \%$ of the HPV $18,77.8 \%$ of the HPV $33,83,3 \%$ of the HPV53 and $100 \%$ of the HPV31 positive cases regressed during the follow up period of 1 year (see Figure 8).

\begin{tabular}{cccccc}
\hline HPV 16 & HPV 58 & HPV 18 & HPV 33 & HPV 53 & HPV 31 \\
\hline $50 \%$ & $72,7 \%$ & $76,9 \%$ & $77,8 \%$ & $83,3 \%$ & $100 \%$ \\
\hline
\end{tabular}

Table 5. Choi et al, Remission of Cytoactiv L1 positive cases within 1 year in relation to the HPV type

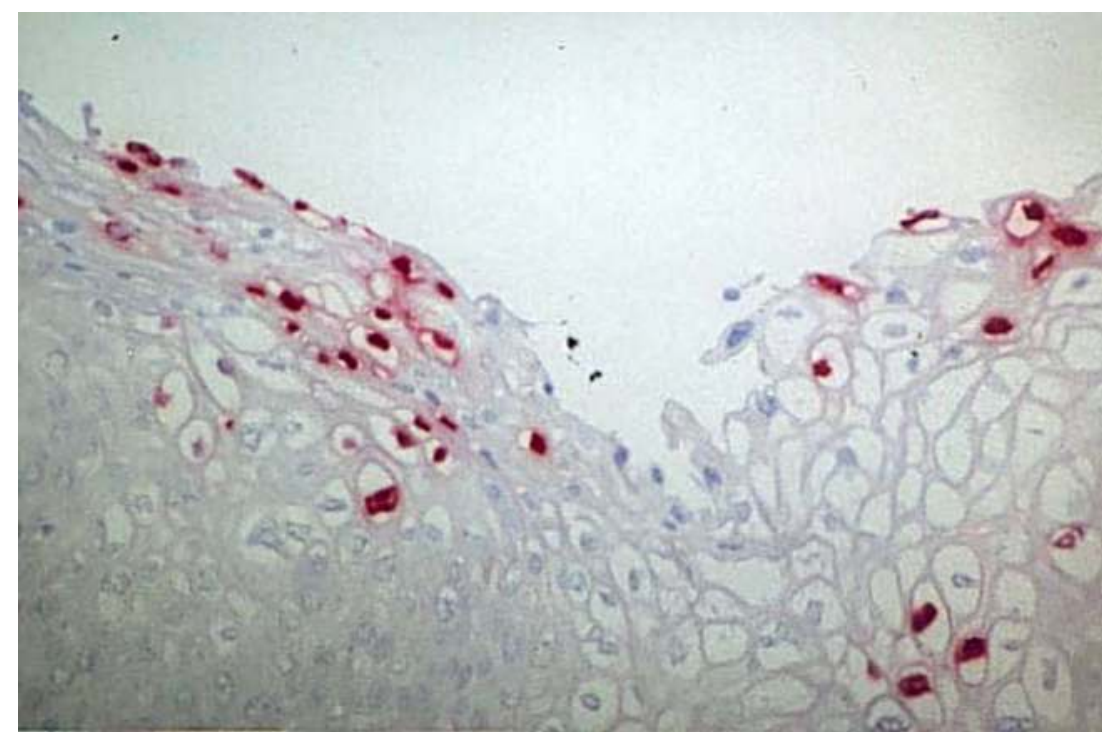

Figure 8. HPV16 / L1+CIN1 - according to Choi et al. regress in 50\% of the cases within 12 month.

In contrast to all other studies Galgano et al. [17] asked if HPV L1 detection, as a stand alone marker, could be a useful diagnostic, but not prognostic, tool.

As HPV specific protein L1 is only detectable in HPV positive lesions. HPV negative CIN lesions have to be L1 negative, because the virus is absent.

According to Hilfrich/Hariri and Negri et al L1 negativ cases are mixtures of HPV associated and non HPV associated CIN lesions, especially analysing CIN1.

HPV positive (p16 positive) but L1 negative lesions are high risk lesions whereas on the other hand HPV negative (p16 negative) and L1 negative lesions are 'no risk' lesion or as Negri mentioned could be classified as 'no evidence of CIN'. 


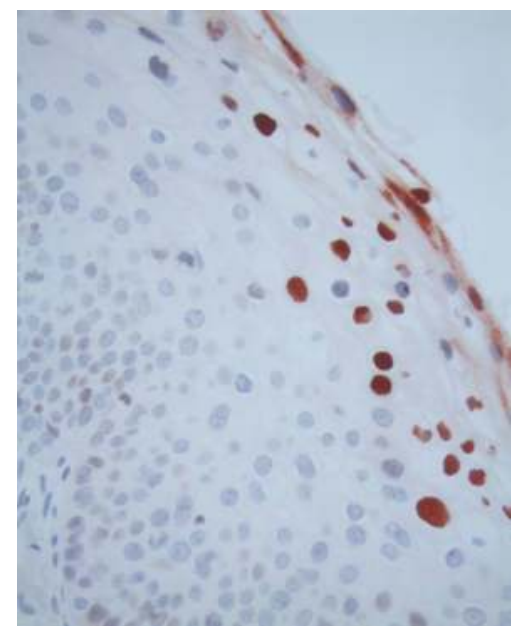

Figure 9. $L 1+C I N 2$

Not surprisingly mixing and not differentiating the L1 negative 'high risk' and the L1 negative 'no risk' entities have to result in 'disappointing' results because remission and progression of the lesions are observed equally.

\subsection{The combination of $\mathrm{L} 1$ / p16 in cytological samples}

In the meantime the combination of L1 and p16 has been investigated on cytological samples by Ungureanu and colleagues [30] as well. As expressed in different phases of cervical carcinogenesis, the authors expected that p16 and L1 are potentially promising markers of progression risk of LSIL. The combination of p16 and L1 capsid protein immunostaining in LBC appears to be useful for an early diagnosis of precancerous lesions and for an appropriate clinical attitude.

Consistent with the previous data they found that expression of L1 capsid protein could be observed in $33.33 \%$ of ASC-US cases, $50 \%$ of LSILs, $18.51 \%$ of HSILs. No positive cases were found in the group of SCC, thus indicating that L1 capsid protein expression tends to decline with increasing severity of the lesions.

\section{Discussion}

\subsection{L1 negative dysplastic lesions as proof of a non-productive, but deregulated life cycle}

As already described a tight communication between the virus and the host cell is of critical importance for the viral life cycle. On the one hand it is strictly linked to the epithelial cell differentiation, on the other hand HPV need to modulate the proliferation / differentiation 
status of the host cells to allow replication in 'non dividing cells' and the maturation of new infectious virus particles.

As long as the L1 capsid protein can be detected within the nucleus of dysplastic cells the virus was successful in this 'walk on the edge'. Despite of all viral activities the cells are still in the condition to allow the normal, productive life cycle of the Human Papilloma Viruses.

L1 capsid protein negative dysplasias, however, are due to this virally induced cellular deregulation processes no longer capable to produce virions.

A shift from a productive HPV infection towards a non-productive or precancerous lesion has occurred.

The reasons for this event are multifarious since the differentiation dependent expression of L1 is controlled at multiple levels. A block at any of the following steps, such as transcription (integration and / or methylation of the DNA), post-transcriptional processing and translation, could be responsible for the loss of L1 capsid protein.

\subsection{Transcriptional control}

\subsubsection{Loss of L1 due to integration of the HPV genome}

Integration of the viral DNA is considered to be of critical importance for the progression from CIN to cancer, since the frequency of HPV-16 viral integration increase in parallel with the severity of cervical lesions.

During the integration process of the HPV genome into the host chromosome a linearization of the ring - shaped, episomal viral DNA is required. It's easy to imagine, that this non directed event is regularly associated with a deregulation of the strictly controlled episomal DNA. As a consequence of the integration process alterations of the control region and loss or disruption of HPV specific proteins like the early and late proteins can be observed [31].

Even if the L1 gene is not affected directly, the integration of the virus with loss of transcriptional control by E2 results in over expression of E6 and E7 leading to immortalization and transformation of the cells [32].

As a result, the epithelial host cell remains in the cell cycle and increasingly becomes genetically instable without being able to run its differentiating program.

L1 genes can functionally be inactivated afterwards too, as a result of mutation, gene deletion and insertion as well as DNA methylation so that no capsid protein will be produced anymore (discussed later).

A dysmaturational autonomous tumor emerges in the host epithelium; a 'point of no return' is crossed.

But for the background that many of the HPV-associated cancers do not even carry any integrated viral genome [31], [33] additional mechanisms have to exist to block L1 expression. In the meantime a discussion started if integration is the initial step towards cervical cancer, or maybe only the consequence of the E6/E7 induced genetic instability of the host cells. 


\subsubsection{Loss of $L 1$ in precancerous lesions with an episomal HPV genome}

Control of gene expression by epigenetic modification of distinct DNA sequences is a fundamental biological process, which affects for example embryonic development, cellular differentiation and others.

One important mechanism, affecting the chromatin conformation, is the methylation of DNA, specifically at cystidine-guanidine $(\mathrm{CpG})$ dinucleotides. Methylated $\mathrm{CpG}$ dinucleotides bind repressors, which alter the conformation of nucleosomes through their interaction with histone deacetylases in a manner unfavourable to transcription [34].

In the meantime it's known that epigenetic mechanisms play a major role in the transcription of the HPV genome as well [35], [36].

Several reports showed that the HPV genome is differentially methylated during progression from simple infected to transformed cells.

Alterations were observed particularly in the control region, and the L1 and L2 gene in high grade precancer and invasive cancer. These observations lead to the suggestion that the lack of expression of these genes may be attributed at least in part to increasing methylation of the respective parts of the viral genomes.

Kalantari et al. for example reported that methylation exceeds $50 \%$ in the case of some CpG dinucleotides within the L1 gene [37].

In addition E2 expression seems to be strictly linked to the differentiation process from normal to malignant cells, indirectly affecting L1 expression as well. Vinokurova showed that E2 binding sites are highly methylated in undifferentiated cells, inhibiting E2-binding, and demethylation at the E2 binding sites occurs in association with the cell differentiation only [38].

That means different mechanisms are existing to prevent L1 mRNA transcription.

\subsection{Post transcriptional control}

Once the transcription of the late mRNA was successful, additional mechanisms have been reported that are able to control or block the L1 capsid protein expression.

\subsubsection{Control of the stability of late $m R N A$}

Mori et al. [39] showed that RNA instability elements are within the L1 and L2 coding mRNAs of HPV16, which function in undifferentiated cells. Although the mechanism for RNA destabilization are still subject of further investigations this mechanism could be important for L1 expression.

\subsubsection{Nuclear export of late $m R N A s$,}

Koffa et al. [40] reported that the L1 mRNA of HPV16 was retained in the nucleus in undifferentiated W12 epithelial cells, suggesting that the nuclear export of late mRNAs was 
inhibited in the dividing cells, thus preventing translation of the L1 protein in the cytoplasm. The factor(s) mediating the nuclear export of late mRNAs has not been identified yet [41].

\subsection{Translational control of late mRNA}

Last but not least the rare codon usages found in L1 and L2 might also contribute to the inhibition of late gene translation [42]. In terminally differentiated cells, the altered expression ratios of tRNA species could compensate for the inhibitory effect of the rare codon usages [43].

All these steps could be of critical importance for L1 capsid protein expression. As indicated a lot of questions are still remaining and need to be answered in the future. Most probably not only a single control mechanism is responsible for the oberserved loss of L1 capsid proteins.

\section{Immune response against L1 capsid proteins}

\subsection{L1 positivity and clinical remission}

The immune system developed special innate and adaptive immune mechanisms to recognize and fight against foreign agents that invade our body.

Sometimes these methods are ineffective especially when the agent uses mechanisms to evade the immune system, like HPV is doing.

Since the HPV infection remains located in the epithelium, mucosal ulceration is a prerequisite for antigen contact with the immune cells in the stroma and in addition to a sufficiently high antigen dose, an efficient immune response, against HPV, also requires supporting mediators.

However, HPV itself has own characteristics also due to its route of infection that protect it from access of the immune system.

The HPV infection does not cause a systemic spreading of the infection by means of a viraemia and the infected epithelia are not destroyed. Thus, any inflammatory tissue reaction supporting the immune response is suppressed, and the virus material is only released on the epithelial surface which is poor in immune defence cells and distant from immune centers.

Finally, the virus itself express only very low levels of viral protein, suppresses the release of cytokines from epithelia and intraepithelial antigen-presenting Langerhans' cells and can suppress the expression of histocompatibility antigens required for the interaction of epithelial cells and immune cells. The E7 and E6 proteins are involved in this inhibition [44], [45].

Consequently, one could envision that in this setting an efficient transfer of antigen from HPV infected keratinocytes to the antigen presenting cells (APC) is not triggered.

Nevertheless a successful immune response to genital HPV infection is established in almost all cases. But the time required for clearance of high risk types, particulary HPV16, averages 8-14 month, which is considerably longer than 5-6 months needed for clearance of low risk types [44]. 


\subsection{The role of the $\mathrm{L} 1$ capsid protein in immune recognition}

The only fully accessible antigen sources in the earlier stage of virally induced SIL to promote an activation of the immune system are free viral particles consisting of $360 \mathrm{~L} 1$ capsid proteins.

Therefore it seems not to be surprising, that a clinical remission of the lesion is observed regularly if the L1 capsid protein is detectable in the dysplastic cells.

To generate an effective virus specific immune response the virus particles have to be detected by the antigen presenting cells (APC) of squamous epithelium, the Langerhans cells (LC) or Dendritic cells (DC), and armed effector cells, has to migrate back to the infected site, and destroy the infected keratinocytes leading to a spontaneous clinical remission of the lesion.

If such immunologic activation mechanisms are functional, they are quite effective since with about $20 \%$ the malignant potential for these L1-positive lesions, irrespective of the dysplasia being cytologically mild or moderate, is low.

On the other hand it is still not clear how L1 specific cytotoxic T-cells could be able to destroy the HPV reservoir in the basal cell layer to cause clinical remission, since the L1 capsid protein is only detectable in terminally differentiated cells.

In analogy to the basal cells it was shown for the L1 capsid protein negative C3 cell line, that these cells are sensitive to L1 specific cytotoxic T-cells [46]. The only explanation seems to be a L1 expression level in these cells (as well as the basal cells), lower than the detection limit used for analysis. As described earlier L1 mRNA is detectable in the nucleus of undifferentiated cells.

A second explanation for the clinical remission of L1 capsid protein positive dysplasias could be, that the viral capsids work as a kind of abjuvance, only triggering the cell mediated response to the early proteins, principally E2 and E6, which are thought to be important for lesion regression [47].

Nevertheless it was shown recently by Mehlhorn et al. [48] that the detection of antibodies against HPV16 L1 in the serum is always associated with clinical remission, if the L1 capsid protein is detectable in the smear of HPV high risk positive mild and moderate dysplasias. These L1 specific serum antibodies shouldn't be able to fight against the HPV infected cells, but it shows that a L1 specific activation of the immune system is of critical importance for the clearance of the HPV infection.

\subsection{Progression of L1 capsid protein positive cases}

An ineffective immune response maybe promoted by HLA incompatibilities, factors contributing to cervical cancer like tobacco smoking or the coexistence of dysplasias of different grade in the transformation zone, possibly reflecting a mixture of L1-positive and L1-negative lesions with different progressive potentials may be the reasons for a progression of some L1positive intraepithelial lesions.

In addition Yang et al [49] identified several mutant HPV16 L1 isolates carrying genes encoding proteins that neither assemble nor activate VLP-dependent innate and adaptive immune 
responses. They concluded that this may represent an additional mechanism of the evasion of innate immune recognition during cervical carcinogenesis.

\subsection{L1 negative dysplasias and progression to CIN3+}

Absence of L1 capsid protein, as the only fully accessible antigen sources in the earlier stage of virally induced SIL, leads to the situation that the viral immune escape mechanisms are maintained and the dysplastic cells, unnoticed by the immune system, proceed in the process of malignant transformation.

With more than $80 \%$ in cytology and more than $90 \%$ for CIN1/2 the malignant potential of the L1-negative dysplasias is exceedingly high, similar to what is expected for a true precancerous lesion.

The differentiation dependent loss of the L1 stimulus may lead to a local 'lack of immunity' further supporting the virally induced alterations.

These may lead to additional disorders of cell cycle regulation at transcriptional, translational and genomic levels thus resulting in a progression of the early precursor lesions to CIN3+. [49]- [51].

\subsection{L1 negative dysplasias and clinical remission}

Reasons for the extremely rare cases of clinical remission of L1-negative cases ( $\sim 5-10 \%)$ are most likely due to sampling errors with absence of L1 expressing cells in the sample or expression levels below the detection limit of the highly sensitive immunochemical assay, as reported for the C3 cell line [46].

\section{Summary}

To treat or not to treat that remains the last question, that has to be answered for women with abnormal Pap smears at the end of the cervical cancer prevention program.

As step 3 of the traditional programs it is common sense that excisional or ablative treatment of the cervical tissue is needed in women diagnosed with precancer.

A statement that is easy to agree on, but difficult to follow since mild, moderate and severe dysplasias or the histological equivalents CIN1, CIN2, CIN3 are mixtures of distinct biological stages resulting in different clinical outcomes and neither cytological follow up, colposcopy nor HPV DNA testing could indicate whether a remission or progression of the precursor lesion to invasive cancer will occur.

The good news is that the ratio of remission or transient HPV infection of the one hand, and progression or precancer on the other hand is moving to precancer with the severity of the lesion. 
But even CIN3 is not uniform in being precancer. Ostor reported that $30 \%$ of these cases will show a spontaneous remission if untreated. Nevertheless we agree that a treatment is warranted.

Accepting CIN2 as the clinical threshold for treatment moves us towards a higher degree of overtreatment, increasing in parallel the potential harms on reproductive outcomes for fertile women, including preterm delivery and low-birth-weight infants with life long fatal disabilities.

As shown HPV L1 detection with Cytoactiv is an objective standard to optimize the clinical management of women with abnormal Pap smears.

The data published over the last decade shows uniform that HPV-L1 detection allows identifying transient HPV infections and precancerous lesions within the group of HPV highrisk positive early dysplastic lesions (mild and moderate dysplasia) see Figure 10.

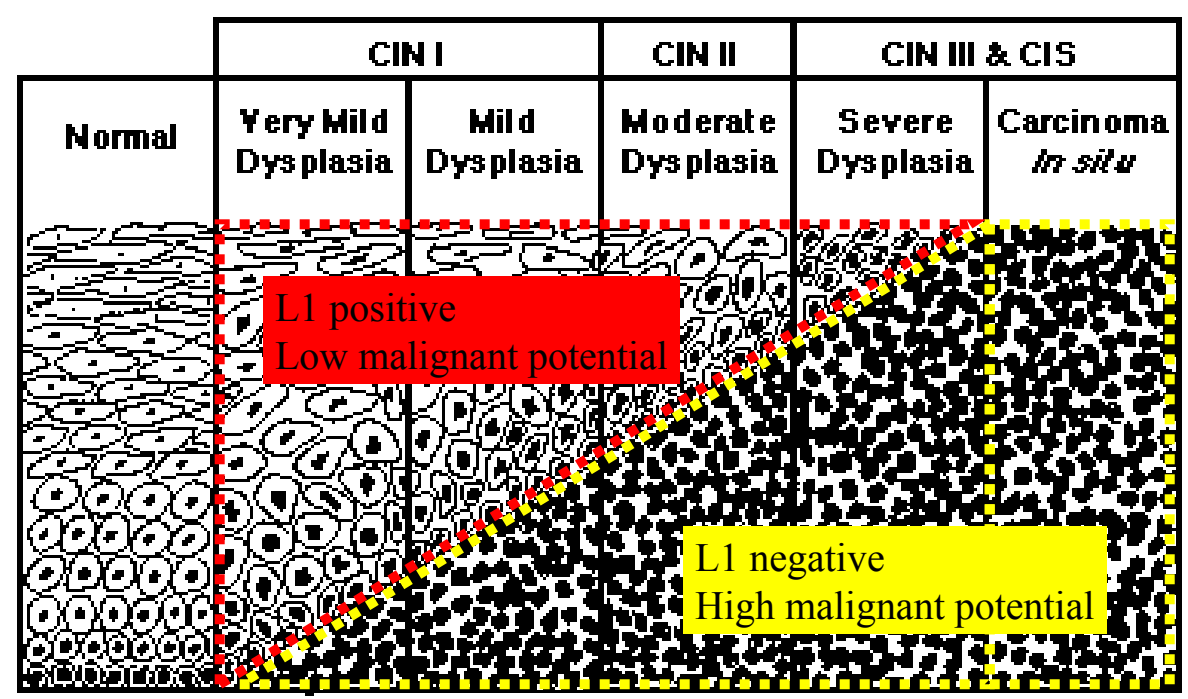

Figure 10. Histologically, CIN grading is based upon the proportion of the surface epithelium composed of undifferentiated cells characteristic of the basal layer. Increasing grade is associated with a progressive loss of epithelial maturation. L1 detection allows to identify the different progressive potentials of transient HPV infection (red) and precancer (yellow).

As summarized in Tables $2-4,75 \%$ of the L1 negative LSIL and $94,2 \%$ of the L1 negative HSIL progressed to CIN3. Using CIN1 and CIN2 lesions with $83,9-96,4 \%$ the results are compareable.

These high progression rates of HPV-L1 negative mild and moderate dysplasia emphasizes the precancerous nature of these lesions. Only 5-25\% of these lesions regress spontaneously a rate even better than what is accepted for treatment of CIN3, but years before the severe dysplasia arise. 
As stated by different authors a close follow-up with colposcopy and histological evaluation is advisable and removal of these lesions should be considered.

On the other hand the low malignant potential of HPV-L1 positive cases indicates transient HPV infection, or true 'low grade lesions'.

Only 13,1\% of the L1 positive LSIL, and 34,6\% of the L1 positive HSIL progressed to CIN3, typically thresholds justifying 'a watch and wait strategy' with cytological follow-up.

Integrating the promising serological HPV L1 antibody rapid test into this procedure seems to be able to improve this data further, thus preventing overtreatment especially for women in their reproductive age.

Only in case of persistence of the L1 positive lesion a colposcopy should be performed.

At the end of the day a combination of cytology, colposcopy, HPV DNA determination and HPV L1 detection offers a unique possibility to increase the benfits of cervical cancer screening programs, by reducing the potentials harms.

\section{Author details}

Ralf Hilfrich

Cytoimmun Diagnostics GmbH Pirmasens, Germany

\section{References}

[1] Ferlay J, Shin HR, Bray F, Forman D, Mathers C and Parkin DM. GLOBOCAN 2008 v1.2, Cancer Incidence and Mortality Worldwide: IARC CancerBase No. 10.Lyon, France: International Agency for Research on Cancer; 2010. Available from: http:// globocan.iarc.fr.

[2] Meisels A, Fortin R : Condylomatous lesions of the cervicx and vagina. I. Cytologic patterns. Acta Cytol 1976, 20 : 505-509

[3] Meisels A, Fortin R, Roy M : Condylomatous lesions of the cervic. II. Cytologic, colposcopic and histopathologic study. Acta Cytol 1977, $21: 379-390$

[4] Gissmann L, zur Hausen H : Partial characterization of viral DNA from human genital warts (Condylomata acuminata). Int J Cancer 1980, 25 : 605-609

[5] WHO/ICO information centre on Human Papilloma Virus (HPV) and Cervical Cancer, www.who.int/hpvcentre 
[6] Papanicolaou GN; Traut HF. Diagnosis of uterine cancer by the vaginal smear. New York, Commonwealth Fund, 1943.

[7] Riotton G, Christopherson WM, Lunt R. Cytology of the Female Genital Tract. International Histological Classification of Tumours No. 8, World Health Organisation, Geneva, 1973.

[8] Solomon D, Davey D, Kurman R et al. The 2001 Bethesda System: terminology for reporting results of cervical cytology. JAMA 2002, 287 (16): 2114-9.

[9] Wagner D. Munich nomenclature II for gynaecologic cytodiagnosis. Acta Cytol 1990; 34: 900-901.

[10] Ostor A. Natural History of Cervical Intrepithelial Neoplasia: A critical review, Int J Gynecol Pathol 1993; 12(2): 186.

[11] Richart RM, Natural history of cervical intraepithelial neoplasia. Clin Obstet Gynecol $1967 ; 10: 748-784$

[12] Kyrgiou M, Koliopoulos G, Martin-Hirsch P, Arbyn M, Prendiville W, Paraskevaidis E. Obstetric outcomes after conservative treatment for intraepithelial or early invasive cervical lesions: systematic review and meta-analysis. Lancet 2006; 367:489-98.

[13] Soergel P, Makowski E, Makowski L, Schippert C, Hillemanns P. What are the costs of conisation when considering pregnancy - associated complications ? Geburtsh Frauenheilk 2011;71: 199-204).

[14] Zur Hausen H, Papillomaviruses and cancer: from basic studies to clinical application. Nat Rev Cancer. 2002; 2 : 342-350.

[15] Schiller JT, Day PM, Kines RC. Current understanding of the mechanism of HPV infection. Gynecol Oncol. 2010 Jun;118(1 Suppl):S12-7.

[16] Doorbar J. Papillomavirus life cycle organization and biomarker selection. Dis Markers 2007;23(4):297-313.

[17] Galgano MT, Castle PE, Atkins KA, Brix WK, Nassau SR, Stoler MH. Using biomarkers as objective standards in the diagnosis of cervical biopsies. Am J Surg Pathol, 2010 Aug; 34(8): 1077-1087.

[18] Melsheimer P, Kaul S, Dobeck S, Bastert G, Immunocytochemical detection of human papillomavirus high risk L1 capsid proteins in LSIL and HSIL as compared with detection of HPV L1 DNA. Acata Cytol. 2003; 47 : 124-128.

[19] Griesser H, Sander H, Hilfrich R, Moser B, Schenck U, Correlation of immunochemical detection of HPV L1 capsid protein in Pap smears with regression of high risk HPV DNA positive mild/moderate dysplasia. Anal Quant Cytol Histol, 2004;26; 241-245.

[20] Rauber D, Mehlhorn G, Fasching PA, Beckmann MW, Ackermann S. Prognostic significance of the detection of the human papillomavirus L1 protein in smears of mild 
to moderate cervical intraepithelial lesions. Eur J Obstet Gynecol Reprod Biol 2008 Oct;140(2):258-62. Epub 2008 Jul 14.

[21] Scheidemantel T, Simmerman K, Ji X, Dolar S, Brainard J, Tubbs R, Hilfrich R, Yang B. 2008. Expression pattern of HPV L1 capsid protein in PAP tests: a potential biomarker in risk assessment for high grade SIL lesion. Abstract Ann. M. Am. Soc. of Cytopathology

[22] Hilfrich R, Weiss A, Griesser H, Use of the ThinPrep ${ }^{\circledR}$ Imager for evaluation of slides stained immunocytochemically with Cytoactiv ${ }^{\circledR}$. Presentation ICC2010, Edinburgh.

[23] Griesser H, Sander H, Walczak C, Hilfrich R. HPV vaccine protein L1 predicts disease outcome of high-risk HPV+ early dysplastic lesions. Am J Clin Pathol 2009 Dec; 132(6):840-5.

[24] Stemberger-Papic S, Vrdoljak-Mozetic D, Ostojic DV, Rubesa-Mihaljevic R, Manestar M, Evaluation of the HPV L1 capsid protein in prognosis of mild and moderate dysplasia of the cervix uteri. Coll Antropol 2010, 34, 2 : 419-423.

[25] Lee SJ, Lee AW, Kim TJ et al. Correlation between immunocytochemistry of human papilloma virus L1 capsid protein and behavior of low-grade cervical cytology in Korean women. Journal of Obstetrics and Gynaecology Research 2011 Sep;37(9):1222-8.

[26] Mehlhorn G, Obermann E, Negri G, Bubendorf L, Mian Chr, Koch M, Sander H, Simm B, Lütge M, Banrevi Zs, Weiss A, Gieri C, Hilfrich R, Beckmann M, Griesser H, HPV L1 detection discriminates cervical precancer from transient HPV infection - a prospective international multicenter study. Nature - Modern Pathology in press.

[27] Negri G, Bellisano G, Zannoni GF et al. p16 and HPV immunohistochemistry is helpful for estimating the behaviour of low grade dysplastic lesions of the cervix uteri. Am J Surg Pathol 2008 Nov;32(11):1715-20.

[28] Hilfrich R, Hariri J. Prognostic relevance of HPV L1 capsid protein detection within mild to moderate dysplastic lesions of the cervix uteri in combination with a second biomarker p16. Anal Quant Cytol Histl 2008 Apr;30(2):78-82.

[29] Choi YS, Kang WD, Kim SM et al. Human Papillomavirus L1 Capsid Protein and Human Papillomavirus Type 16 as Prognostic Markers in Cervical Intraepithelial Neoplasia 1. Int J of Gynecological Cancer 2010 Feb;20(2):288-93.

[30] Ungureanu C, Socolov D, Anton G, Mihailovici MS, Teleman S, Immunocytochemical expression of p16ink4a and HPV L1 capsid proteins as predictive markers of the cervical lesions progression risk. Rom J Morphol Embryol. 2010;51(3):497-503.

[31] Vinokurova S, Wentzensen N, Kraus I et al., Type-dependent integration frequency of human papillomavirus genomes in cervical lesions. Cancer Res 2008; 68: 307-313. 
[32] Romanczuk H, Howley PM, Disruption of either the E1 and E2 regulatory gene of human papillomavirus type 16 increase viral immortalization capacity. Proc Natl Acad Sci USA, 89 : 3159-3163.

[33] Pett M, Coleman N, Integration of high-risk human papilloma virus a key event in cervical carcinogenesis?, J Pathol 212: 356-367.

[34] Goll MG, Bestor TH. Eukaryotic cytosine methyltransferases. Annu Rev Biochem. 2005;74:481-514.

[35] Kulis M, Esteller M, DNA methylation and cancer. Adv. Genet 2010, 70 : 27-56.

[36] Badal V, Chuang LS, Tan EH, Badal S, Villa LL et al., CpG methylation of human papillomavirus type 16 DNA in cervical cancer cell lines and in clinical specimen : Genomic hypomethylation correlates with carcinogenic progression. J Virol 77: 6227-6234.

[37] Kalantari M, Calleja-Macias IE, Tewari D, Hagmar B, Lie K, Barrera-Saldana HA, Wiley DJ, Bernard HU. Conserved methylation patterns of human papillomavirus type 16 DNA in asymptomatic infection and cervical neoplasia. J Virol. 2004 Dec;78(23): $12762-72$.

[38] Vinokurova S, von Knebel Doeberitz M, Differential Methylation of the HPV 16 upstream regulatory region during epithelial differentiation and neoplastic transformation. PloS ONE 2011, 6 (9), 24451-24464.

[39] Mori S, Ozaki S, Yasugi T, Yoshikawa H, Taketani Y, Kanda T, Inhibitory cis-element-mediated decay of human papillomavirus type 16 L1-transcript in undifferentiated cells. Mol Cell Biochem 2006, 288, 47-57

[40] Koffa MD, Graham SV, Takagaki Y, Manley JL, Clements JB, The human papillomavirus type 16 negative regulatory RNA element interacts with three proteins that act at different posttranscriptional levels. Proc Natl Acad Sci USA, 2000, 97, 4677-4682.

[41] Zhao X, Rush M, Schwartz S, Identification of an hnRNA A1-dependent splicing silencer in the human papillomavirus type $16 \mathrm{~L} 1$ coding region that prevents premature expression of the late L1 gene. J Virol 2004, 78, 10888-10905.

[42] Gu W, Li M, Zhao WM, Fang NX, Bu S, Frazer IH, Zhao KN, tRNASer(CGA) differentially regulates expression of wildtype and codon-modified papillomavirus L1 genes. Nucl Acids Res 2004, 32, 4448-4461.

[43] Fang NX, Gu W, Ding J, Saunders NA, Frazer IH, Zhao KN, Calcium enhances keratinocyte differentiation in vitro to differentially regulate expression of papillomavirus authentic and codon modified L1 genes. Virology 2007, 365, 187-197.

[44] Stanley M, Immune responses to human papillomvirus. Vaccine 2006, 24 (Suppl.1): $16-22$ 
[45] Scott M, Nakagawa M, Moscicki AB, Cell-mediated immune response to human papillomavirus infection. Clin Diagn Lab Immunol 2001, 8 : 209-220.

[46] Ohlschläger P, Osen W, Dell K, Faath S, Garcea RL, Jochmus I, Müller M, Pawlita M, Schäfer K, Sehr P, Staib C, Sutter G, Gissmann L. Human papillomavirus type 16 L1 capsomeres induce L1-specific cytotoxic T lymphocytes and tumor regression in C57BL/6 mice. J Virol. 2003 Apr;77(8):4635-45.

[47] van Poelgeest MI, Nijhuis ER, Kwappenberg KM, Hamming IE, Wouter Drijfhout J, Fleuren GJ, van der Zee AG, Melief CJ, Kenter GG, Nijman HW, Offringa R, van der Burg SH. Distinct regulation and impact of type 1 T-cell immunity against HPV16 L1, E2 and E6 antigens during HPV16-induced cervical infection and neoplasia. Int J Cancer. 2006 Feb 1;118(3):675-83.

[48] Mehlhorn G, Koch M, Hilfrich R, Beckmann M, HPV16-L1-specific antibody rapidtest improves the prognostic significance of Cytoactiv. HPV2011, Berlin.

[49] Yang R, Wheeler CM, Chen X et al. Papillomavirus capsid mutation to escape dentritic cell dependent innate immunity in cervical cancer. J Virol 2005; 79 (11): 6741-6750.

[50] Choo KB, Lee HH, Pan CC et al. Sequence duplication and internal deletion in the integrated human papillomavirus type 16 genome from cervical carcinoma. J Virol 1998; 62: 1659-66.

[51] Icenogle JP, Clancy KA, Lin SY. Sequence variation in the capsid protein genes of human papillomavirus type 16 and type 31. Virology 1995; 214: 664-669. 